\title{
(C) \\ Evaluation and comparison of the new swept source OCT-based IOLMaster 700 with the IOLMaster 500
} OPEN ACCESS

\author{
Ahmet Akman, Leyla Asena, Sirel Gür Güngör
}

Department of Ophthalmology, Başkent University, Faculty of Medicine, Ankara, Turkey

\section{Correspondence to} Professor Ahmet Akman, Department of Ophthalmology, Baskent University Faculty of Medicine, Fevzi Çakmak Caddesi, Bahçelievler, Ankara 06490, Turkey; ahmetakman@ hotmail.com

Received 2 September 2015 Revised 13 November 2015 Accepted 22 November 2015 Published Online First 16 December 2015

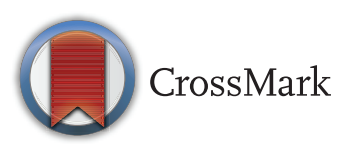

To cite: Akman A, Asena $L$, Güngör SG. Br J Ophthalmol 2016;100:1201-1205.
ABSTRACT

Purpose To compare the measurements and failure rates obtained with a new swept source optical coherence tomography (OCT)-based biometry to IOLMaster 500.

Setting Eye Clinic, Baskent University Faculty of Medicine, Ankara, Turkey.

Design Observational cross-sectional study and evaluation of a new diagnostic technology.

Methods 188 eyes of 101 subjects were included in the study. Measurements of axial length (AL), anterior chamber depth (ACD), corneal power (K1 and K2) and the measurement failure rate with the new Zeiss IOLMaster 700 were compared with those obtained with the IOLMaster 500. The results were evaluated using Bland-Altman analyses. The differences between both methods were assessed using the paired samples $t$ test, and their correlation was evaluated by intraclass correlation coefficient (ICC).

Results The mean age was $68.32 \pm 12.71$ years and the male/female ratio was 29/72. The agreements between two devices were outstanding regarding $\mathrm{AL}$ $(I C C=1.0), A C D(I C C=0.920), K 1 \quad(I C C=0.992)$ and $K 2$ (ICC $=0.989)$ values. IOLMaster 700 was able to measure ACD AL, K1 and K2 in all eyes within highquality SD limits of the manufacturer. IOLMaster 500 was able to measure ACD in 175 eyes, whereas measurements were not possible in the remaining 13 eyes. AL measurements were not possible for 17 eyes with IOLMaster 500. Nine of these eyes had posterior subcapsular cataracts and eight had dense nuclear cataracts.

Conclusions Although the agreement between the two devices was excellent, the IOLMaster 700 was more effective in obtaining biometric measurements in eyes with posterior subcapsular and dense nuclear cataracts.

\section{INTRODUCTION}

The advancement of new technologies in intraocular lens (IOL) design and surgical techniques has increased the expectations of patients for exceptional postoperative vision without any refractive correction. The correct measurement of axial length (AL) and corneal power are crucial for the calculation of IOL power and achievement of the desired postoperative refraction. ${ }^{1}$ Modern optical biometry devices use either partial coherence interferometry (PCI) or optical low coherence reflectometry (OLCR) to measure several variables including AL. In addition they can measure keratometry $(\mathrm{K})$, anterior chamber depth (ACD), lens thickness (LT) and horizontal white-to-white (WTW) corneal diameter using different approaches. $^{2}$ Moreover, the built-in software in these devices provides more accurate IOL power calculation and multiple choices of IOL formulas. ${ }^{3}$ The IOLMaster (Carl Zeiss Meditec AG, Jena, Germany) is the gold standard of modern optical biometry devices. It uses the principle of PCI to obtain AL with high precision. ${ }^{4} 5$ Several reports have shown the accuracy of IOLMaster (IOLMaster or IOLMaster 500) for IOL calculation in routine and complicated cataract cases. ${ }^{6-9}$ However, measurements can be difficult or unreliable with corneal opacities, macular diseases, advanced and posterior subcapsular (PSC) cataracts, vitrectomised eyes or poor fixation for PCI or OLCR based devices. ${ }^{10} 11$

The IOLMaster 700 (Carl Zeiss Meditec AG, Jena, Germany), which is the first swept source optical coherence tomography (OCT) based biometry, was introduced recently. It enables OCT imaging and visualisation across the entire length of the eye. It provides an image-based measurement, allowing the surgeon to view the complete longitudinal section of the eye. Therefore, it may identify irregular eye geometries, such as lens tilt. Additionally, imaging of the fovea may alert the observer to insufficient fixation during measurements. It also uses telecentric keratometry for corneal power measurements, similar to the IOLMaster 500. Briefly, it was introduced as an advantageous diagnostic device in an attempt to improve refractive results after cataract surgery.

The purpose of this study was to compare the agreement between IOLMaster 500 and IOLMaster 700 values for $\mathrm{ACD}, \mathrm{AL}$ and keratometry. Measurement failure rates with both devices were also recorded and compared.

\section{PATIENTS AND METHODS}

This observational cross-sectional study comprising patients with cataract was performed at the Başkent University Faculty of Medicine, Department of Ophthalmology, Ankara, Turkey. The study was performed according to the Declaration of Helsinki. The University's ethics committee approved the study design and protocol. The patients were fully informed about the purpose of the study, after which they provided informed consent.

Patients who were either diagnosed for the first time or who were under follow-up for cataract were included in the study. Patients were recruited during a 3-month period between April and June 2015. All patients were examined by the same physician (AA) and in addition to routine ophthalmological examinations, cataract types were recorded as nuclear, cortical or PSC according to Lens Opacities Classification III scoring system (LOCS $\quad$ III). ${ }^{12}$ Biometric measurements were 
performed before pupillary dilatation in all patients. Patients who were unable to cooperate and fixate adequately during the measurements, with advanced macular problems like cystoid macular oedema or elevated scars, additionally whom had previous ocular surgery and irregular corneal surfaces were not included in the study. Corneal surface was investigated by Scheimpflug system (WaveLight Allegro Oculyzer; WaveLight AG, Erlangen, Germany). AL, ACD and corneal power (K1 (flattest axis); K2 (steepest axis of corneal curvature $90^{\circ}$ apart from flat axis)), and failure rates for both instruments were compared.

\section{Instruments}

IOLMaster 500 uses the PCI principle for AL measurements, a six-point telecentric technique for $\mathrm{K}$ readings and an imagebased slit lamp system for ACD measurements. It can also measure WTW distance. However, LT and central corneal thickness (CCT) measurements are not available on IOLMaster 500. With this device, five consecutive measurements of $\mathrm{AL}$ and $\mathrm{ACD}$, and three consecutive keratometry measurements were performed. A signal to noise ratio (SNR) value for AL readings is calculated. The manufacturer does not recommend the use of the measurements with SNR value less than two. Quality of keratometry testing can be verified by visually checking the quality of the readings.

IOLMaster 700 automatically takes swept source OCT scans and measures AL, along with CCT, ACD and LT. AL measurements are the average values of three scans in each of six meridians. For $\mathrm{K}$ readings, three average $\mathrm{Ks}$, each of five single measurements are taken and the final average $\mathrm{K}$ readings are calculated. On the IOLMaster 700, the operator can see the whole scan image and visually check the eye geometry and axis of the measurements; also the foveal scan checks the correct fixation by the patient. SD values of the ACD, LT and AL measurements are calculated and the device warns the operator of low-quality results if the $\mathrm{SD}$ for $\mathrm{ACD}>0.021 \mathrm{~mm}$, for $\mathrm{LT}>0.038 \mathrm{~mm}$ and for $\mathrm{AL}>0.027 \mathrm{~mm}$.

\section{Biometry measurement technique}

Biometric measurements were performed using IOLMaster 500 and IOLMaster 700 consecutively by the same technician for the first 100 eyes and the order was reversed for the remaining eyes. The quality control criteria for both devices were used in accordance with the above-mentioned manufacturer recommendations. If measurements were not possible in accordance with limits, they were recorded as measurement failures for either device. The same examiner, who was trained according to the manufacturer's recommendations, performed all tests.

All IOLMaster 700 scans were checked by the same surgeon (AA) for foveal scans to ensure the correct axis measurements and the scan was repeated if the patient was not fixating correctly.

\section{Statistical analysis}

The required sample size was calculated using the following formula: $n=\left[\left(2 \times t_{f, 0,95} \times S D\right) / C I\right]^{2}$ with $t_{n-1 ; 1-\alpha / 2}=1,7$ (quantile of $\mathrm{t}$ distribution for expected sample size $\mathrm{n}>50$ and $\alpha=0.05$; $\mathrm{SD} ; \mathrm{CI}){ }^{2}$ The anticipated SDs were based on the results from preliminary study data for initial power analysis. ${ }^{13}$

The results were evaluated using intraclass correlation coefficients (ICCs), Bland-Altman plots and paired samples t tests. Left and right eyes were included in the subsequent analysis, as most of the variation in the ocular variables considered in this study is found within rather than between the left and right eyes

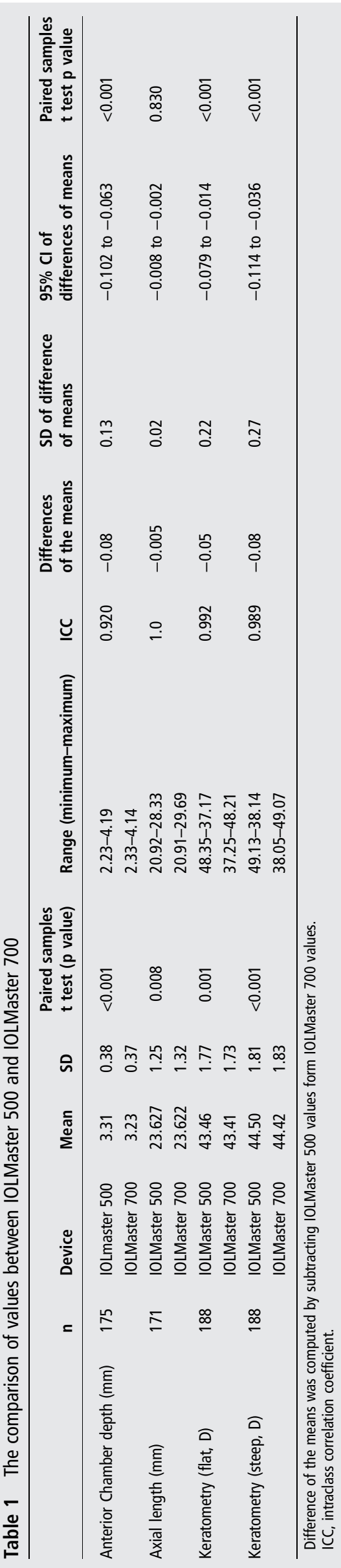




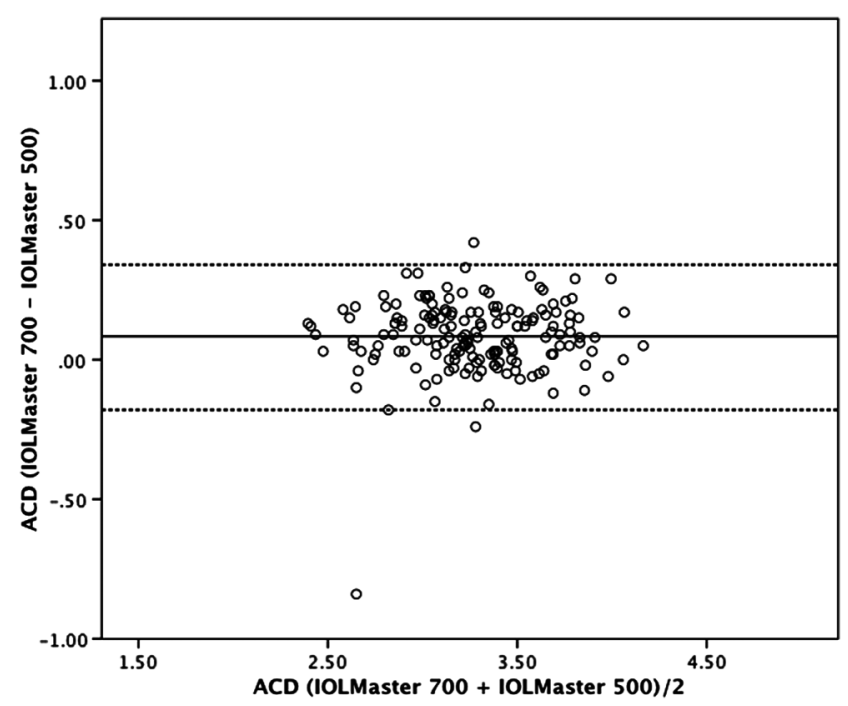

Figure 1 Bland-Altman plot for anterior chamber depth (ACD) comparing IOLMaster 700 with IOLMaster 500 . The $95 \%$ limits of agreement were -0.33 to $0.17 \mathrm{~mm}$. Dotted lines, $\pm 1.96 \mathrm{SD}$.

(analysis of variance p values $>0.05$ for AL, ACD, K1 and K2). Including both eyes in the study was also in accordance with similar previous studies. $^{2} 14$

Statistical analysis was performed using SPSS software (V.22.0 for Mac, SPSS Inc, Chicago, Illinois, USA).

\section{RESULTS}

Data from 188 eyes of 101 patients were included in the study (male/female: 29/72). The sample size proved statistically to be sufficient to determine and prove the data for ACD, AL, K1, K2 with desired precision and probability. The mean age of the patients was 68.32 \pm 12.71 (range 24-81) years. One hundred and thirty-five eyes $(71.8 \%)$ had nuclear cataracts, 18 eyes (9.5\%) had cortical cataracts and 35 eyes $(18.6 \%)$ had PSC cataracts. Best corrected Snellen visual acuity ranged from finger counting to $0.9(0.51 \pm 0.23)$.

Table 1 summarises the ACD, AL, K1, K2 results for IOLMaster 500 and IOLMaster 700. Bland-Altman plots for comparisons between IOLMaster 500 and IOLMaster 700 are presented in figures $1-4$.

Although the agreements between the two devices were outstanding regarding $\mathrm{AL}, \mathrm{ACD}, \mathrm{K} 1$ and $\mathrm{K} 2$ values, paired sample $\mathrm{t}$ tests showed statistically significant differences among ACD, K1 and K2 measurements. These differences are quite small for clinical relevance and table 2 summarises the differences between two measurements for clinically acceptable values for $\mathrm{ACD}, \mathrm{AL}, \mathrm{K} 1$ and $\mathrm{K} 2$.

IOLMaster 700 was able to measure ACD, AL, K1 and K2 in all eyes within high-quality SD limits of the manufacturer. IOLMaster 500 was able to measure ACD in 175 eyes, whereas measurements were not possible in the remaining 13 eyes. Nine of these 13 eyes had ACD values longer than $4.1 \mathrm{~mm}$ (IOLMaster 700 measurement data). AL measurements were not possible for 17 phakic eyes with IOLMaster 500. Nine of these eyes had PSC and eight had dense nuclear cataracts.

\section{DISCUSSION}

Satisfactory refractive results after IOL implantation depends on optimal biometry. Accurate AL, ACD and K readings are essential for all biometric formulas to calculate the IOL power required for the desired results. With the common usage of

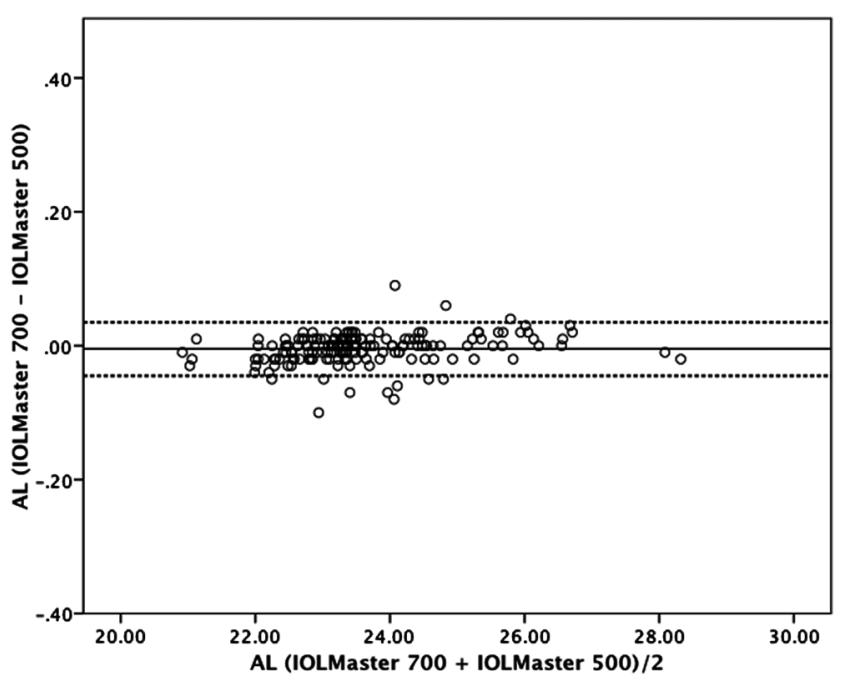

Figure 2 Bland-Altman plot for axial length (AL) comparing IOLMaster 700 with IOLMaster 500 . The $95 \%$ limits of agreement were -0.045 to $0.035 \mathrm{~mm}$. Dotted lines, $\pm 1.96 \mathrm{SD}$.

toric and multifocal IOLs, accurate biometry has become more important than ever to reach the refractory goals that patients expect. The most common preventable sources of error are incorrect AL measurements and keratometry readings. ${ }^{15}$

IOLMaster was the first optical biometer available for commercial usage and its accuracy and repeatability made it the gold standard of biometry for many years. ${ }^{16}{ }^{17}$ Other optical biometry devices became available in the last few years. All these devices depend on patient fixation to a target. Poor fixation can be one of the reasons for AL measurement errors. Lens problems, dense vitreous opacities or foveal problems can also cause errors in optical biometry. In addition, with PSC and dense cataracts, the optical biometers available today can fail to take adequate measurements. ${ }^{11} 18$

Swept source OCT based IOLMaster 700 provides an imagebased measurement, allowing the surgeon to view the complete longitudinal section of the eye. Therefore, it may identify irregular eye geometries, such as lens tilt, and imaging of the

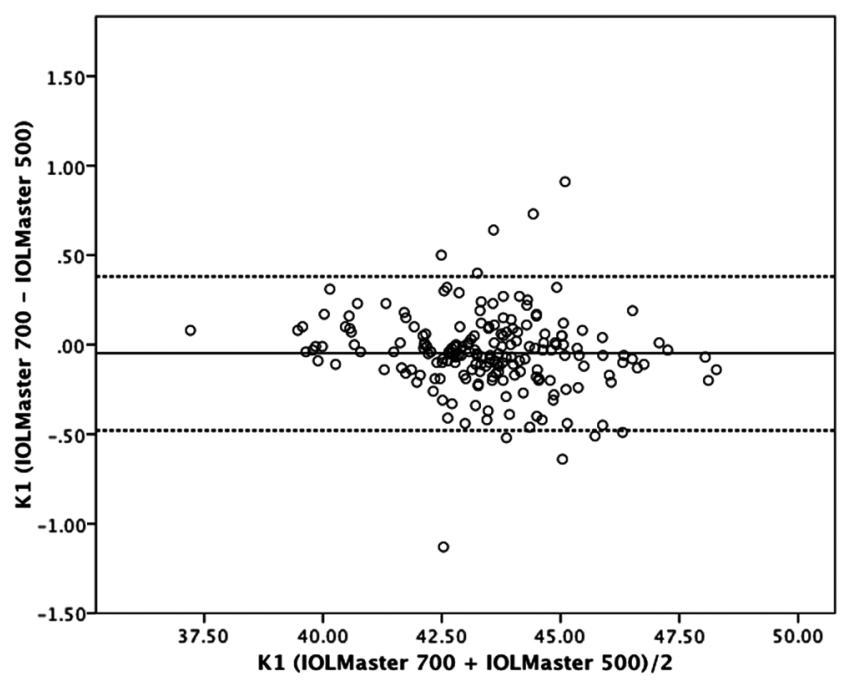

Figure 3 Bland-Altman plot for K1 value comparing IOLMaster 700 with IOLMaster 500 . The $95 \%$ limits of agreement were -0.48 to 0.38 D. Dotted lines, \pm 1.96 SD. 


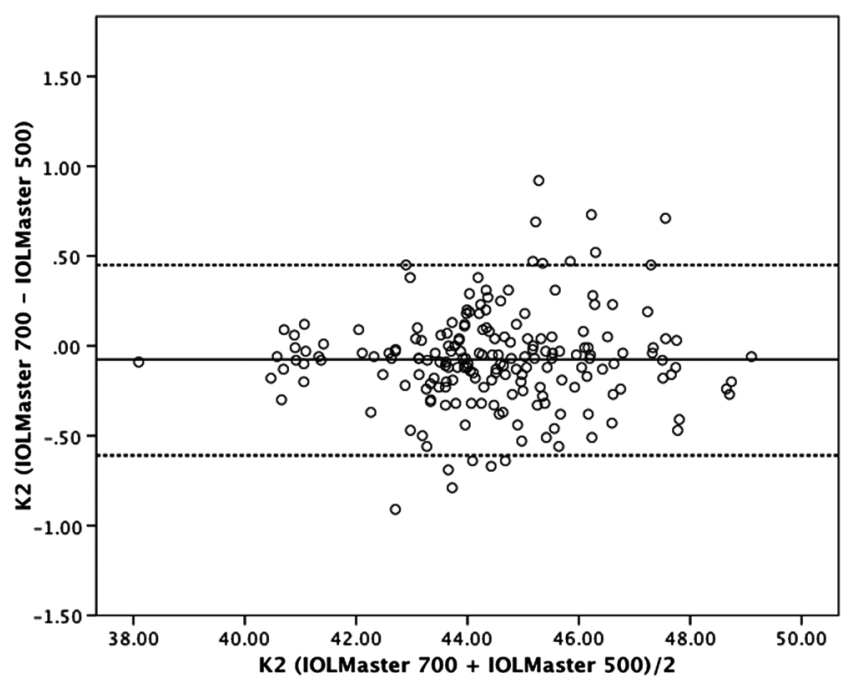

Figure 4 Bland-Altman plot for K2 value comparing IOLMaster 700 with IOLMaster 500 . The $95 \%$ limits of agreement were -0.61 to 0.45 D. Dotted lines, \pm 1.96 SD.

fovea may alert the observer to insufficient fixation during measurements. This may allow for a more accurate performance of IOL power calculations and better refractive outcomes. Furthermore, the swept source OCT technology has the additional advantage of extremely rapid data acquisition, including the ability to measure the AL along six different axes.

In this study, we aimed to compare the performance of IOLMaster 700 with the gold standard IOLMaster 500. Regarding the AL measurements, IOLMaster 700 showed an incredible correlation with IOLMaster 500 with a ICC value of 1. The mean measurement difference among the 188 eyes measured was $0.005 \mathrm{~mm}(\mathrm{SD}=0.02)$. These results show that both devices measure the AL in nearly the same way.

In eyes with PSC, IOLMaster 500 and other optical biometers have higher failure rates and immersion ultrasound (US) biometry was the only option until today. ${ }^{11} 18$ Clinically, PSC cataracts are well known to cause more visual disability compared with other cataract types, even in low grades. As the opacities are located nearer to the nodal point of the lens, more light rays will be affected. This can be one of the reasons for measurement failures in PCI or OLCR based optical biometries. ${ }^{18}$ Using swept source OCT technology may overcome these problems and this could be the main reason for the higher acquisition rate for $\mathrm{AL}$ measurements with IOLMaster 700 in the presence of PSC or dense cataracts. In our study, IOLMaster 700 was able to measure AL in all 188 phakic eyes, including the PSC and dense nuclear cataracts. However, IOLMaster 500 failed to acquire measurements in 9 of the 35 PSC cases and in 8 dense cataracts.

Measurement failure rate of IOLMaster 500 can be reduced by using a higher number of measurements and selectively using the ones with better quality, a method described by Hill et al. ${ }^{19}$ But these methods are time consuming, require a higher number of measurements and the measurements need to be reviewed by an experienced and skilful operator. ${ }^{19}$ For routine clinical practice, these methods were not feasible in our clinical setting and we did not employ them.

ACD measurements of IOLMaster 700 also correlated very highly with the IOLMaster 500. IOLMaster 500 does not measure ACD with PCI. The principal that IOLMaster 500 uses for ACD measurements is based on an optical section through the anterior chamber by means of a slit-illumination system with subsequent image assessment. It can be prone to operator errors and this method cannot measure the ACD in pseudophakic eyes. $^{20} 21$ However, IOLMaster 700 uses swept source OCT images and is able to measure ACD, CCT and LT from the single OCT image that is aligned with the visual axis of the eye. Our results show that ACD measurements with IOLMaster 700 are highly correlated with IOLMaster $500 \quad(\mathrm{ICC}=0.940)$. However, when we compared the mean differences of these groups, we saw that IOLMaster 700 measured ACD $0.08 \mathrm{~mm}$ shorter than IOLMaster 500. Although these values are very small for clinical significance, this finding is statistically significant $(p<0.001)$. As only fourth-generation formulas like Holliday II and Olsen use ACD data, it will not affect the thirdgeneration formula results and this $0.08 \mathrm{~mm}$ difference will cause negligible IOL power difference for the fourth-generation formulas. Again, the ACD measurement failures were observed only with IOLMaster 500. For 13 eyes in which IOLMaster 500 failed to obtain a measurement, nine eyes had ACD measured $>4.1 \mathrm{~mm}$ with the IOLMaster 700. In previous studies, ACD measurements obtained with IOLMaster 500 have been reported and compared with different US-based and optically based instruments, with variable results. ${ }^{22-24}$ ACD values were reported to be higher ${ }^{25}$ compared with ultrasonographic techniques in some reports and lower ${ }^{26}$ in others. A number of reasons may be proposed for these differences. The IOLMaster 500 may not measure the axial ACD because the slit source is projected from the temporal side. Measuring the anterior chamber off centre may result in an error. Another possible cause is the inclusion of keratometry in calculating the ACD. Both of the above-mentioned potential sources of error are

Table 2 The differences in measurements between IOLMaster 500 and IOLMaster 700; n (\%)

Keratometry measurements $(n=188)$

\begin{tabular}{|c|c|c|c|c|c|c|c|}
\hline \multicolumn{2}{|c|}{$\begin{array}{l}\text { Anterior chamber } \\
\text { depth }(n=175)\end{array}$} & \multicolumn{2}{|c|}{ Axial length $(n=188)$} & \multicolumn{2}{|l|}{ K1 } & \multicolumn{2}{|l|}{ K2 } \\
\hline Depth (mm) & n (\%) & Length (mm) & n (\%) & $\begin{array}{l}\text { Keratometry } \\
\text { measurements (D) }\end{array}$ & n (\%) & $\begin{array}{l}\text { Keratometry } \\
\text { measurements (D) }\end{array}$ & n (\%) \\
\hline$<0.1 \mathrm{~mm}$ & $90(52)$ & No difference & $43(24)$ & $<0.5 \mathrm{D}$ & 181 (96) & $<0.5 \mathrm{D}$ & $173(92)$ \\
\hline$<0.2 \mathrm{~mm}$ & 154 (88) & $<0.02 \mathrm{~mm}$ & $102(56)$ & $<1 D$ & $188(100)$ & $<1 \mathrm{D}$ & $188(100)$ \\
\hline \multirow[t]{4}{*}{$<0.3 \mathrm{~mm}$} & $175(100)$ & $<0.03 \mathrm{~mm}$ & $150(82)$ & & & & \\
\hline & & $<0.05 \mathrm{~mm}$ & $165(91)$ & & & & \\
\hline & & $<0.10 \mathrm{~mm}$ & $177(96)$ & & & & \\
\hline & & $<0.15 \mathrm{~mm}$ & $188(100)$ & & & & \\
\hline
\end{tabular}


eliminated in IOLMaster 700, since it uses swept source OCT images for the calculation of ACD.

Keratometric measurements are the second important source of error for refractive outcomes. Both devices use a distance-independent telecentric keratometry system. In our study, nearly all of the keratometry measurements with both instruments were within 0.5 (D) for steep and flat meridians. Besides, the correlation coefficients for flat meridian $(\mathrm{ICC}=0.992)$ and steep meridian $(\mathrm{ICC}=0.989)$ show that both instruments measure corneal power with similar accuracy. Although very important for toric IOL calculations, axis measurements are not used by most of the current biometric formulas for spheric IOL power calculations and so we did not include them in the current paper.

With ICC close to 1 , both devices are expected to give very similar IOL measurements in eyes with good fixation and no additional ocular problems, which is the case for most of the routine cataract cases we observe in developed countries. The main advantage of swept source OCT-based biometry like IOLMaster 700 becomes evident in patients with poor fixation, irregular eye geometries and denser cataracts.

In this study we found that the IOL power for SRK/T, Holiday 1, Hoffer Q and Haigis derived by both devices were quite similar, and highly correlated. The mean difference was less than $0.10 \mathrm{D}$ for all the formulas, less than the increment in the IOL power step $(0.50 \mathrm{D})$. Although both devices did not have a clinically significant impact on IOL power, the chosen IOL formula should be considered. If the WTW measurement is required in a formula, such as in the Holladay 2, the result may be different.

As a result, the agreement between the two devices was outstanding regarding $\mathrm{AL}, \mathrm{ACD}, \mathrm{K} 1$ and $\mathrm{K} 2$ values. However, the IOLMaster 700 was more effective in obtaining biometric measurements in eyes with PSC and dense nuclear cataracts compared with IOLMaster 500. The ability of the new swept source OCT-based biometry to detect the fixation pattern, decentration or tilt of the lens and the tomographic visualisation of the complete longitudinal section of the eye including the fovea helps to eliminate potential sources of error and, therefore, to optimise refractive outcomes in cataract surgery.

Contributors AA: substantial contributions to the conception or design of the work, or the acquisition, analysis or interpretation of data; drafting the work or revising it critically for important intellectual content; final approval of the version published. LA: substantial contributions to the conception or design of the work, or the acquisition, analysis or interpretation of data; drafting the work or revising it critically for important intellectual content. SGG: drafting the work or revising it critically for important intellectual content; final approval of the version published; agreement to be accountable for all aspects of the work in ensuring that questions related to the accuracy or integrity of any part of the work are appropriately investigated and resolved.

Competing interests None declared.

Patient consent Obtained.

Ethics approval Baskent University.

Provenance and peer review Not commissioned; externally peer reviewed.

Open Access This is an Open Access article distributed in accordance with the Creative Commons Attribution Non Commercial (CC BY-NC 4.0) license, which permits others to distribute, remix, adapt, build upon this work non-commercially, and license their derivative works on different terms, provided the original work is properly cited and the use is non-commercial. See: http://creativecommons.org/ licenses/by-nc/4.0/

\section{REFERENCES}

1 Olsen T. Calculation of intraocular lens power: a review. Acta Ophthalmol Scand 2007;85:472-85.

2 Rohrer $\mathrm{K}$, Frueh BE, Wälti R, et al. Comparison and evaluation of ocular biometry using a new noncontact optical low-coherence reflectometer. Ophthalmology 2009; 116:2087-92.

3 Wang J-K, Chang S-W. Optical biometry intraocular lens power calculation using different formulas in patients with different axial lengths. Int J Ophthalmol 2013;6:150-4.

4 Vogel A, Dick HB, Krummenauer F. Reproducibility of optical biometry using partial coherence interferometry: intraobserver and interobserver reliability. J Cataract Refract Surg 2001;27:1961-8.

5 Fontes BM, Fontes BM, Castro E. Intraocular lens power calculation by measuring axial length with partial optical coherence and ultrasonic biometry. Arq Bras Oftalmol 2011;74:166-70.

6 Hsieh Y-T, Wang I-J. Intraocular lens power measured by partial coherence interferometry. Optom Vis Sci 2012;89:1697-701.

7 Kunavisarut $\mathrm{P}$, Poopattanakul $\mathrm{P}$, Intarated $\mathrm{C}$, et al. Accuracy and reliability of IOL Master and A-scan immersion biometry in silicone oil-filled eyes. Eye 2012;26:1344-8.

8 Roessler GF, Dietlein TS, Plange N, et al. Accuracy of intraocular lens power calculation using partial coherence interferometry in patients with high myopia. Ophthalmic Physiol Opt 2012;32:228-33.

9 Olsen T. Improved accuracy of intraocular lens power calculation with the Zeiss IOLMaster. Acta Ophthalmol Scand 2007;85:84-7.

10 Tehrani M, Krummenauer F, Blom E, et al. Evaluation of the practicality of optical biometry and applanation ultrasound in 253 eyes. J Cataract Refract Surg 2003;29:741-6.

11 Freeman $\mathrm{G}$, Pesudovs $\mathrm{K}$. The impact of cataract severity on measurement acquisition with the IOLMaster. Acta Ophthalmol Scand 2005;83:439-42.

12 Chylack LT, Wolfe JK, Singer DM, et al. The Lens Opacities Classification System III. The Longitudinal Study of Cataract Study Group. Arch Ophthalmol 1993;111:831-6.

13 Akman A, Güngör SG. Comparison of new IOLMaster 700 swept source biometry system with the IOLMaster 500 optical biometry. Paper presented at ESCRS Meeting, 5-9 September 2015; Barcelona, Spain.

14 Jasvinder S, Khang TF, Sarinder KK, et al. Agreement analysis of LENSTAR with other techniques of biometry. Eye (Lond) 2011;25:717-24.

15 Jin GJ, Crandall AS, Jones JJ. Intraocular lens exchange due to incorrect lens power. Ophthalmology 2007;114:417-24.

16 Chen YA, Hirnschall N, Findl O. Evaluation of 2 new optical biometry devices and comparison with the current gold standard biometer. J Cataract Refract Surg 2011;37:513-7.

17 Kaswin G, Rousseau A, Mgarrech M, et al. Biometry and intraocular lens power calculation results with a new optical biometry device: comparison with the gold standard. J Cataract Refract Surg 2014;40:593-600.

18 McAlinden C, Wang Q, Pesudovs K, et al. Axial length measurement failure rates with the IOLMaster and Lenstar LS 900 in eyes with cataract. PLOS ONE 2015;10:128-929.

19 Hill W, Angeles R, Otani T. Evaluation of a new IOLMaster algorithm to measure axial length. J Cataract Refract Surg 2008;34:920-4

20 Kriechbaum K, Findl O, Kiss B, et al. Comparison of anterior chamber depth measurement methods in phakic and pseudophakic eyes. J Cataract Refract Surg 2003:29:89-94.

21 Chang SW, Yu CY, Chen DP. Comparison of intraocular lens power calculation by the IOLMaster in phakic and eyes with hydrophobic acrylic lenses. Ophthalmology 2009;116:1336-42.

22 Elbaz U, Barkana Y, Gerber Y, et al. Comparison of different techniques of anterior chamber depth and keratometric measurements. Am J Ophthalmol 2007;143:48-53

23 Uçakhan ÖÖ, Akbel V, Biyikli Z, et al. Comparison of corneal curvature and anterior chamber depth measurements using the manual keratometer, Lenstar LS 900 and the Pentacam. Middle East Afr J Ophthalmol 2013;20:201-6.

24 Huang J, Liao N, Savini G, et al. Comparison of anterior segment measurements with Scheimpflug/Placido photography-based topography system and IOLMaster partial coherence interferometry in patients with cataracts. J Ophthalmol 2014; 540-760.

25 Reddy AR, Pande MV, Finn P, et al. Comparative estimation of anterior chamber depth by ultrasonography, Orbscan II, and IOLMaster. J Cataract Refract Surg 2004:30:1268-71.

26 Santodomingo-Rubido J, Mallen EA, Gilmartin B, et al. A new non-contact optical device for ocular biometry. $\mathrm{Br} J$ Ophthalmol 2002;86:458-62. 